\title{
Half-Sandwich Arene-Osmium(II) Complexes with Phosphinite Ligands
}

\section{Rebeca González-Fernández ${ }^{1}$, Javier Borge ${ }^{2}$, Pascale Crochet ${ }^{1, *}$ and Victorio Cadierno ${ }^{1, *(D)}$}

1 Laboratorio de Compuestos Organometálicos y Catálisis (Unidad Asociada al CSIC), Centro de Innovación en Química Avanzada (ORFEO-CINQA), Departamento de Química Orgánica e Inorgánica, IUQOEM, Facultad de Química, Universidad de Oviedo, Julián Clavería 8, E-33006 Oviedo, Spain; rebeka.g.f@gmail.com

2 Departamento de Química Física y Analítica, Facultad de Química, Universidad de Oviedo, Julián Clavería 8, E-33006 Oviedo, Spain; jborge@uniovi.es

* Correspondence: crochetpascale@uniovi.es (P.C.); vcm@uniovi.es (V.C.); Tel.: +34-985-105076 (P.C.); +34-985-103453 (V.C.)

Received: 17 December 2019; Accepted: 15 January 2020; Published: 17 January 2020 updates

Abstract: The synthesis of a series of arene-osmium(II) complexes containing phosphinite-type ligands, namely, $\left[\mathrm{OsCl}_{2}\left(\eta^{6}-p\right.\right.$-cymene $\left.)\left\{\mathrm{R}_{2} \mathrm{PO}\left(\mathrm{CH}_{2}\right)_{n} \mathrm{Ph}\right\}\right]\left(\mathrm{R}=\mathrm{Ph}, \mathrm{n}=1\right.$ (4a), 2 (4b), 3 (4c); $\mathrm{R}={ }^{\mathrm{i}} \mathrm{Pr}$, $\mathrm{n}=1(5 \mathbf{a}), 2(5 \mathbf{b}), 3(5 \mathbf{c}))$ and $\left[\mathrm{OsCl}_{2}\left(\eta^{6}\right.\right.$-benzene) $\left.\left.{ }_{\{}^{\mathrm{i}} \mathrm{Pr}_{2} \mathrm{PO}\left(\mathrm{CH}_{2}\right)_{2} \mathrm{Ph}\right\}\right](7)$, is presented. All these compounds were characterized by elemental analysis and multinuclear NMR spectroscopy $\left({ }^{31} \mathrm{P}\left\{{ }^{1} \mathrm{H}\right\}\right.$, ${ }^{1} \mathrm{H}$ and $\left.{ }^{13} \mathrm{C}\left\{{ }^{1} \mathrm{H}\right\}\right)$, and the structure of $\left[\mathrm{OsCl}_{2}\left(\eta^{6}-p\right.\right.$-cymene) $\left.\left\{\mathrm{Ph}_{2} \mathrm{PO}\left(\mathrm{CH}_{2}\right)_{3} \mathrm{Ph}\right\}\right](4 \mathrm{c})$ unequivocally confirmed through a single-crystal $\mathrm{X}$-ray diffraction study. Attempts to generate the tethered species $\left[\mathrm{OsCl}_{2}\left\{\eta^{6}: \kappa^{1}(P)-\mathrm{C}_{6} \mathrm{H}_{5}\left(\mathrm{CH}_{2}\right)_{\mathrm{n}} \mathrm{OPR}{ }_{2}\right\}\right]$ by intramolecular exchange of the coordinated arene in 4-5a-c or 7 , upon thermal or MW heating, failed.

Keywords: organometallic compounds; osmium complexes; phosphinite ligands; half-sandwich complexes

\section{Introduction}

We recently described the preparation of the ruthenium(II) complexes $\left[\operatorname{RuCl}_{2}\left\{\eta^{6}: \kappa^{1}(P)\right.\right.$ $\left.\left.\mathrm{C}_{6} \mathrm{H}_{5}\left(\mathrm{CH}_{2}\right)_{\mathrm{n}} \mathrm{OPR}_{2}\right\}\right]\left(\mathrm{R}=\mathrm{Ph},{ }^{i} \mathrm{Pr} ; \mathrm{n}=1,2,3\right.$; see Scheme 1) starting from the corresponding phosphinites $\mathrm{R}_{2} \mathrm{PO}\left(\mathrm{CH}_{2}\right)_{\mathrm{n}} \mathrm{Ph}$ and the chloride-bridged dimers $\left[\left\{\mathrm{RuCl}(\mu-\mathrm{Cl})\left(\eta^{6} \text {-arene }\right)\right\}_{2}\right]$ (arene $=p$-cymene, benzene), along with their structural characterization by single-crystal X-ray diffraction [1].

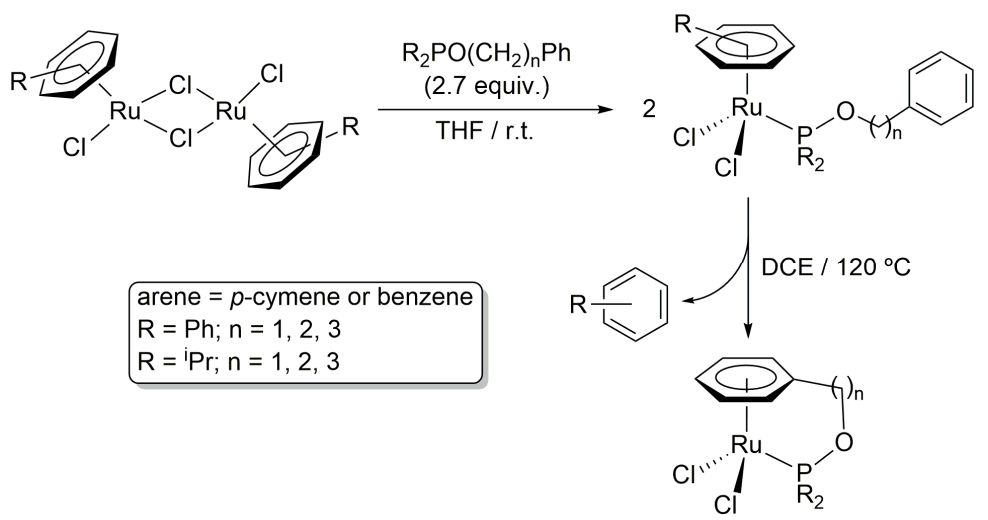

Scheme 1. Route employed for the synthesis of complexes $\left[\mathrm{RuCl}_{2}\left\{\eta^{6}: \kappa^{1}(P)-\mathrm{C}_{6} \mathrm{H}_{5}\left(\mathrm{CH}_{2}\right)_{\mathrm{n}} \mathrm{OPR} \mathrm{R}_{2}\right\}\right]$. 
These compounds are of interest as they represent rare examples of tethered arene-ruthenium(II) complexes incorporating pendant phosphinite donors [2-4], a field largely dominated by the use of arene ligands featuring classical phosphines as the pendant donor group [1,5]. Moreover, we also demonstrated their utility as catalysts for the cross dehydrogenative coupling of hydrosilanes with alcohols [1], a process of relevance since it allows a simple access to useful alkoxysilane reagents [6,7], and with potential application in the field of hydrogen storage and production [8]. As shown in Scheme 1, the synthesis of complexes [ $\left.\mathrm{RuCl}_{2}\left\{\eta^{6}: \kappa^{1}(P)-\mathrm{C}_{6} \mathrm{H}_{5}\left(\mathrm{CH}_{2}\right)_{n} \mathrm{OPR} \mathrm{R}_{2}\right\}\right]$ was accomplished in two steps involving the initial cleavage of the chloride bridges of $\left[\left\{\mathrm{RuCl}(\mu-\mathrm{Cl})\left(\eta^{6} \text {-arene }\right)\right\}_{2}\right]$ by the phosphinites, to generate the corresponding mononuclear adducts $\left[\mathrm{RuCl}_{2}\left(\eta^{6}\right.\right.$-arene $\left.)\left\{\mathrm{R}_{2} \mathrm{PO}\left(\mathrm{CH}_{2}\right)_{n} \mathrm{Ph}\right\}\right]$, and a subsequent intramolecular exchange of the coordinated arene under thermal conditions $\left(120^{\circ} \mathrm{C}\right)$ [1].

On the other hand, tethered osmium complexes are uncommon, and most of the examples currently known involve $\eta^{5}$-cyclopentadienyl-type ligands connected to P- [9,10], N- [10], C- [11], or Sn-donor [12] groups. In fact, the only tethered $\eta^{6}$-arene-osmium(II) derivatives described to date in the literature are compounds $\mathbf{B}$, synthesized by Xia and co-workers from the reactions of the hydride-alkenylcarbyne complex A with allenoates (Scheme 2) [13].

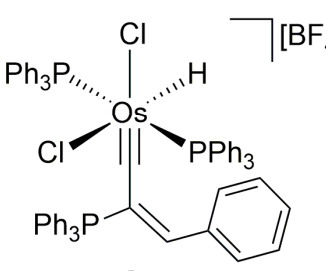

A

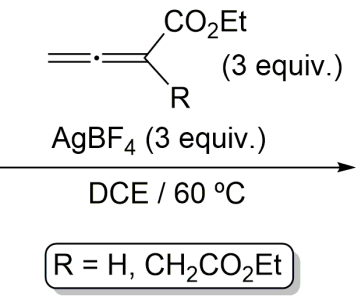

$\mathrm{R}=\mathrm{H}, \mathrm{CH}_{2} \mathrm{CO}_{2} \mathrm{Et}$

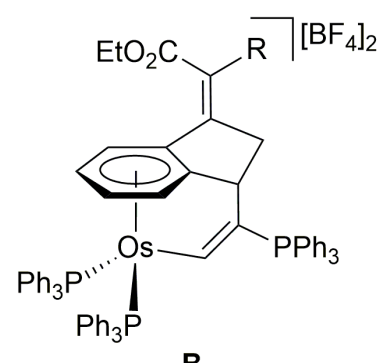

B

Scheme 2. Synthesis of the tethered arene-osmium(II) complexes B.

All these facts prompted us to study the reactivity of the osmium(II) dimers $\left[\left\{\mathrm{OsCl}(\mu-\mathrm{Cl})\left(\eta^{6} \text {-arene) }\right\}_{2}\right]\right.$ (arene $=p$-cymene, benzene) towards phosphinites $\mathrm{R}_{2} \mathrm{PO}\left(\mathrm{CH}_{2}\right)_{\mathrm{n}} \mathrm{Ph}$ as a potential entry to tethered species related to those previously described for ruthenium.

\section{Results and Discussion}

As the starting point of our investigation, we synthesized the mononuclear complexes $\left[\mathrm{OsCl}_{2}\left(\eta^{6}\right.\right.$-p-cymene) $\left.\left\{\mathrm{R}_{2} \mathrm{PO}\left(\mathrm{CH}_{2}\right)_{\mathrm{n}} \mathrm{Ph}\right\}\right]\left(\mathrm{R}=\mathrm{Ph}, \mathrm{n}=1(\mathbf{4 a}), 2(\mathbf{4 b}), 3(4 \mathbf{c}) ; \mathrm{R}={ }^{\mathrm{i}} \mathrm{Pr}, \mathrm{n}=1(5 \mathbf{a}), 2\right.$ (5b), 3 (5c)) by reacting the cymene-osmium dimer $\left[\left\{\mathrm{OsCl}(\mu-\mathrm{Cl})\left(\eta^{6}-p \text {-cymene }\right)\right\}_{2}\right](3)$ with the appropriate phosphinite ligand $\mathrm{R}_{2} \mathrm{PO}\left(\mathrm{CH}_{2}\right)_{n} \mathrm{Ph}(\mathbf{1 - 2 a - c})$. As shown in Scheme 3, the cleavage of the chloride bridges of 3 proceeded quickly and cleanly in tetrahydrofuran at room temperature, affording complexes 4-5a-c in good yields (67-85\%).

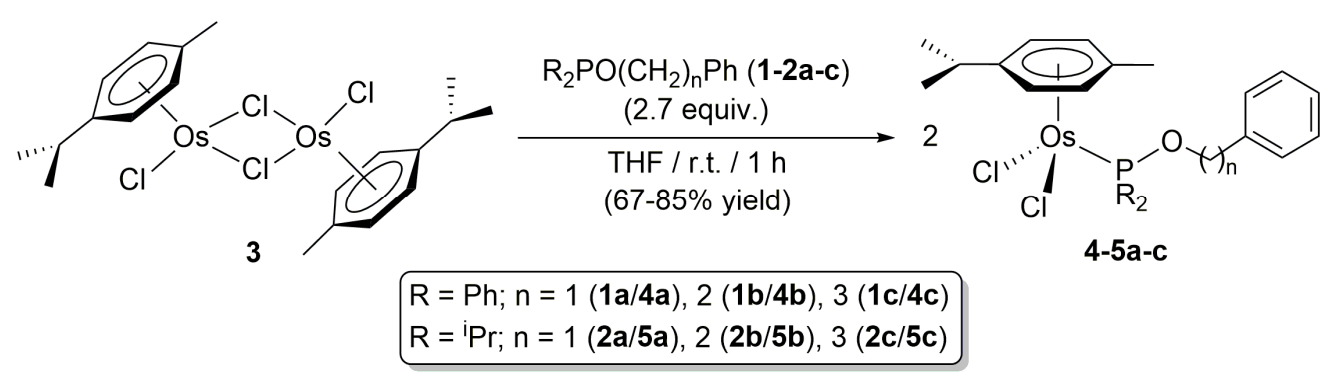

Scheme 3. Synthesis of the osmium(II) complexes $\left[\mathrm{OsCl}_{2}\left(\eta^{6}-p\right.\right.$-cymene $\left.)\left\{\mathrm{R}_{2} \mathrm{PO}\left(\mathrm{CH}_{2}\right)_{n} \mathrm{Ph}\right\}\right](4-5 \mathrm{a}-\mathrm{c})$. 
Compounds $\left[\mathrm{OsCl}_{2}\left(\eta^{6}-p\right.\right.$-cymene $\left.)\left\{\mathrm{R}_{2} \mathrm{PO}\left(\mathrm{CH}_{2}\right)_{n} \mathrm{Ph}\right\}\right]$ (4-5a-c) were isolated as air-stable yellow solids and fully characterized by means of elemental analyses and multinuclear NMR spectroscopy $\left({ }^{31} \mathrm{P}\left\{{ }^{1} \mathrm{H}\right\},{ }^{1} \mathrm{H}\right.$ and ${ }^{13} \mathrm{C}\left\{{ }^{1} \mathrm{H}\right\}$; copies of the spectra are included in the Supplementary Materials file), being the data obtained in complete accord with the proposed formulations. In particular, the ${ }^{31} \mathrm{P}\left\{{ }^{1} \mathrm{H}\right\} \mathrm{NMR}$ spectra confirmed the coordination of the phosphinite ligands to the osmium center by the appearance of singlet resonances shielded 40-50 ppm with respect to those of the uncoordinated phosphinites 1-2a-c (see Table 1). Concerning the ${ }^{1} \mathrm{H}$ and ${ }^{13} \mathrm{C}\left\{{ }^{1} \mathrm{H}\right\}$ NMR spectra, they showed the expected signals for the $\eta^{6}$-coordinated $p$-cymene unit, as well as those of the respective $P$-donor ligand (details are given in the Materials and Methods section). For the former, only two signals for the aromatic $\mathrm{CH}$ proton and carbon atoms of the cymene ring were observed, which is in full accord with the presence of a symmetry plane in the complexes. Interestingly, while the $\mathrm{POCH}_{2}$ carbon in complexes 4a-c resonates as a singlet, a doublet signal was observed for $5 \mathbf{a}-\mathrm{c}\left({ }^{2} J_{\mathrm{PC}}=10-12 \mathrm{~Hz}\right)$. This difference, previously observed in the analogous ruthenium complexes [1], is probably associated with the higher steric requirements of the ${ }^{\mathrm{i}} \mathrm{Pr}$ vs. Ph groups that would affect the corresponding $\mathrm{P}-\mathrm{O}-\mathrm{C}$ bond angle and consequently the $\mathrm{P}-\mathrm{C}$ coupling.

Table 1. ${ }^{31} \mathrm{P}$ chemical shifts for the phosphinite ligands $\mathbf{1 - 2 a - c}$ and the osmium(II) complexes 4-5a-c ${ }^{1}$.

\begin{tabular}{cccc}
\hline Ligand & $\delta_{\mathbf{P}}(\mathbf{p p m})$ & Complex & $\delta_{\mathbf{P}}(\mathbf{p p m})$ \\
\hline $\mathbf{1 a}$ & 114.5 & $\mathbf{4 a}$ & 64.2 \\
$\mathbf{1 b}$ & 126.6 & $\mathbf{4 b}$ & 63.1 \\
$\mathbf{1 c}$ & 112.1 & $\mathbf{4} \mathbf{c}^{2}$ & 62.2 \\
$\mathbf{2 a}$ & 154.5 & $\mathbf{5 \mathbf { a } ^ { 2 }}$ & 103.5 \\
$\mathbf{2 b}$ & 152.3 & $\mathbf{5 b}$ & 103.3 \\
$\mathbf{2 c}$ & 151.4 & $\mathbf{5 c}$ & 102.3
\end{tabular}

${ }^{1}$ Unless otherwise stated, the spectra were recorded in $\mathrm{CDCl}_{3} .{ }^{2}$ Spectra recorded in $\mathrm{CD}_{2} \mathrm{Cl}_{2}$.

Additionally, the structure of $\left[\mathrm{OsCl}_{2}\left(\eta^{6}-p\right.\right.$-cymene $\left.)\left\{\mathrm{Ph}_{2} \mathrm{PO}\left(\mathrm{CH}_{2}\right)_{3} \mathrm{Ph}\right\}\right](4 \mathrm{c})$ was unequivocally confirmed by single-crystal X-ray diffraction. An ORTEP-type view of the molecule, along with selected bond distances and angles, is shown in Figure 1. The osmium atom features the expected pseudo-octahedral three-legged piano-stool geometry, with metrical parameters around the metallic core comparing well with those previously described for the related complexes [OsCl 2 ( $\eta^{6}$ - $p$-cymene) $\left.\left(\mathrm{PPh}_{3}\right)\right][14]$ and $\left[\mathrm{OsCl}_{2}\left(\eta^{6}-p\right.\right.$-cymene $\left.)\left(\mathrm{PPh}_{2} \mathrm{OH}\right)\right][15]$. The Os(1)-P(1) and $\mathrm{P}(1)-\mathrm{O}(1)$ bond distances (2.318(2) and 1.611(4) $\AA$, respectively) are also comparable to those found in the structure of $\left[\mathrm{OsCl}_{2}\left(\eta^{6}-p\right.\right.$-cymene $\left.)\left(\mathrm{PPh}_{2} \mathrm{OH}\right)\right](\mathrm{Os}-\mathrm{P}=2.321(1) \AA$ and $\mathrm{P}-\mathrm{O}=1.603(4) \AA)$ [15]. 


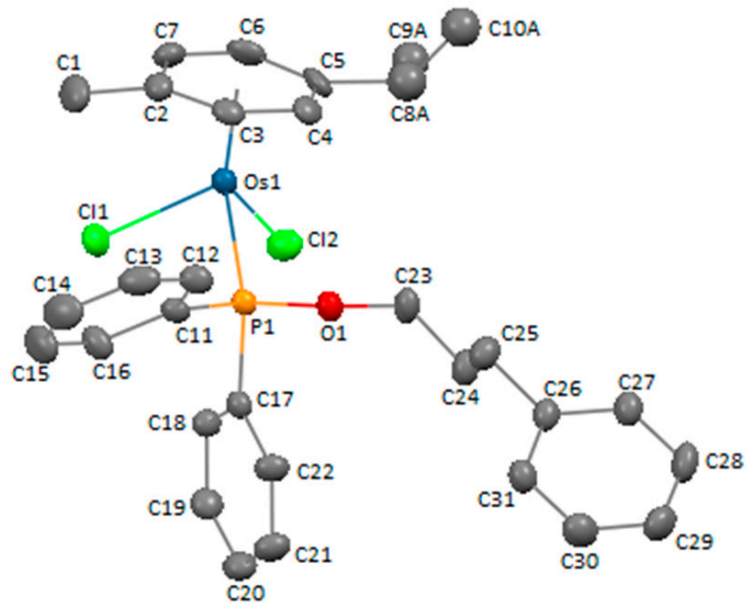

Figure 1. ORTEP-type view of the structure of complex 4c showing the crystallographic labeling scheme. Hydrogen atoms have been omitted for clarity. Thermal ellipsoids are drawn at the $20 \%$ probability level. Selected bond distances ( $⿱$ ) and angles (deg): $\mathrm{Os}(1)-\mathrm{C}^{*}=1.1706(2) ; \quad \mathrm{Os}(1)-\mathrm{Cl}(1)=2.408(2) ; \quad \mathrm{Os}(1)-\mathrm{Cl}(2)=2.406(2) ; \quad \mathrm{Os}(1)-\mathrm{P}(1)=2.318(2)$; $\mathrm{P}(1)-\mathrm{O}(1)=1.611(4) ; \mathrm{P}(1)-\mathrm{C}(11)=1.803(6) ; \mathrm{P}(1)-\mathrm{C}(17)=1.823(6) ; \quad \mathrm{Os}(1)-\mathrm{C}(23)=1.448(7) ;$ $\mathrm{C}^{*}-\mathrm{Os}(1)-\mathrm{P}(1)=127.47(4) ; \mathrm{C}^{*}-\mathrm{Os}(1)-\mathrm{Cl}(1)=125.55(5) ; \mathrm{C}^{*}-\mathrm{Os}(1)-\mathrm{Cl}(2)=127.96(4) ; \mathrm{Cl}(1)-\mathrm{Os}(1)-\mathrm{Cl}(2)=86.83(7)$; $\mathrm{Cl}(1)-\mathrm{Os}(1)-\mathrm{P}(1)=86.26(6) ; \mathrm{Cl}(2)-\mathrm{Os}(1)-\mathrm{P}(1)=89.31(6) ; \mathrm{Os}(1)-\mathrm{P}(1)-\mathrm{O}(1)=114.0(2) ; \mathrm{Os}(1)-\mathrm{P}(1)-\mathrm{C}(11)=111.6(2)$; Os(1)-P(1)-C(17) = 124.2(2); C(11)-P(1)-C(17) = 103.1(3); $\mathrm{C}(11)-\mathrm{P}(1)-\mathrm{O}(1)=98.5(2) ; \mathrm{C}(17)-\mathrm{P}(1)-\mathrm{O}(1)=101.9$ (3); $\mathrm{P}(1)-\mathrm{O}(1)-\mathrm{C}(23)=120.2(4) ; \mathrm{C}^{*}$ denotes the centroid of the $p$-cymene ring $(\mathrm{C}(2), \mathrm{C}(3), \mathrm{C}(4), \mathrm{C}(5), \mathrm{C}(6)$ and $\mathrm{C}(7))$.

Once characterized, compounds 4-5a-c were next evaluated as potential precursors of the corresponding tethered species $\left[\mathrm{OsCl}_{2}\left\{\eta^{6}: \kappa^{1}(P)-\mathrm{C}_{6} \mathrm{H}_{5}\left(\mathrm{CH}_{2}\right)_{\mathrm{n}} \mathrm{OPR} \mathrm{R}_{2}\right\}\right.$. Against our wishes, heating solutions of 4-5a-c in 1,2-dichloethane (DCE) or toluene at $120^{\circ} \mathrm{C}$ did not lead to the intramolecular exchange of the coordinated arene, even after long reaction periods $(24 \mathrm{~h})$. In all the cases, complexes 4-5a-c were recovered unchanged. Application of microwaves irradiation (MW), instead of conventional oil-bath thermal heating, was also unsuccessful. In view of this, we decided to explore the possibility of using related osmium(II) precursors containing benzene instead of $p$-cymene, as, in our previous work with ruthenium, the displacement of the $\eta^{6}$-coordinated benzene ligand proceeded in general much faster than that of the $p$-cymene one (Scheme 1) [1]. To this end, complex $\left[\mathrm{OsCl}_{2}\left(\eta^{6}\right.\right.$-benzene) $\left.\left.{ }_{\{}^{\mathrm{i}} \mathrm{Pr}_{2} \mathrm{PO}\left(\mathrm{CH}_{2}\right)_{2} \mathrm{Ph}\right\}\right]$ (7) was synthesized by reacting dimer [\{OsCl $\left.(\mu-\mathrm{Cl})\left(\eta^{6} \text {-benzene) }\right\}_{2}\right]$ (6) with ${ }^{\mathrm{i}} \mathrm{Pr}_{2} \mathrm{PO}\left(\mathrm{CH}_{2}\right)_{2} \mathrm{Ph}(\mathbf{2 b})$ (see Scheme 4). Due to the poor solubility of 6 , the bridge-splitting reaction required in this case harsher conditions (refluxing in toluene for $24 \mathrm{~h}$ ), and 7 could only be isolated in moderate yield (synthetic details and characterization data are included in the Materials and Methods section). Unfortunately, all attempts to generate $\left[\mathrm{OsCl}_{2}\left\{\eta^{6}: \kappa^{1}(P)-\mathrm{C}_{6} \mathrm{H}_{5}\left(\mathrm{CH}_{2}\right)_{2} \mathrm{OP}^{\mathrm{i}} \mathrm{Pr}_{2}\right\}\right]$ from 7 , upon thermal or MW heating, also failed.
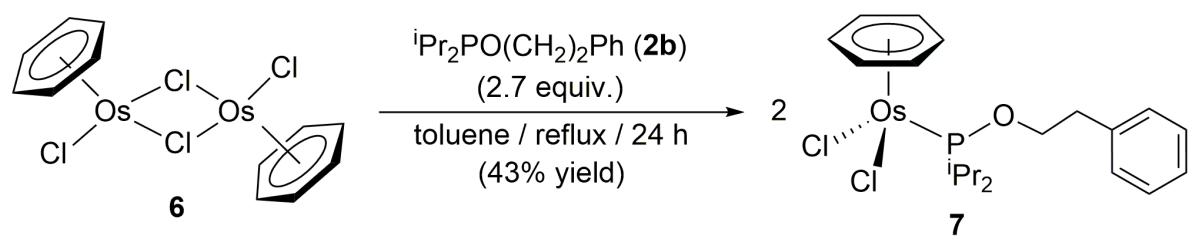

Scheme 4. Synthesis of the osmium(II) complex $\left[\mathrm{OsCl}_{2}\left(\eta^{6}\right.\right.$-benzene $\left.)\left\{{ }^{\mathrm{i}} \mathrm{Pr}_{2} \mathrm{PO}\left(\mathrm{CH}_{2}\right)_{2} \mathrm{Ph}\right\}\right]$ (7). 
The marked differences in reactivity found between the osmium complexes herein synthesized, i.e., 4-5a-c and 7, and their ruthenium counterparts can be ascribed to the higher activation energies associated to ligand substitution processes in osmium vs. ruthenium complexes, a fact well documented in the literature [16-18].

\section{Materials and Methods}

All manipulations were carried out under an inert atmosphere of dry argon employing vacuum-line and Schlenk techniques. Solvents were dried and purified before use, according to standard procedures [19]. The phosphinite ligands 1-2a-c [20,21] and the osmium(II) dimers 3 [22] and 6 [23] were synthesized as described in the literature. A Bruker DPX-300 instrument (Billerica, MA, USA) was employed for NMR measurements (all the spectra were recorded at room temperature). For the ${ }^{13} \mathrm{C}\left\{{ }^{1} \mathrm{H}\right\}$ and ${ }^{1} \mathrm{H}$ NMR chemical shifts, the residual signal of deuterated solvent was employed as reference, while for the ${ }^{31} \mathrm{P}\left\{{ }^{1} \mathrm{H}\right\} \mathrm{NMR}$ ones $85 \% \mathrm{H}_{3} \mathrm{PO}_{4}$ was used as external standard. DEPT experiments were carried out for all the compounds synthesized. Elemental analyses were provided by the Analytical Service of the Instituto de Investigaciones Químicas (IIQ-CSIC) of Seville using a LECO TruSpec CHN analyzer (St. Joseph, MI, USA).

\subsection{General Procedure for the Preparation of Complexes $\left[\mathrm{OsCl}_{2}\left(\eta^{6}\right.\right.$-p-cymene $\left.)\left\{\mathrm{R}_{2} \mathrm{PO}\left(\mathrm{CH}_{2}\right)_{n} \mathrm{Ph}\right\}\right](\mathrm{R}=\mathrm{Ph}$,} $n=1$ (4a), 2 (4b), $3(\mathbf{4} \mathbf{c}) ; R={ }^{i} P r, n=1$ (5a) $\left., 2(5 \mathbf{b}), 3(5 \mathbf{c})\right)$

A suspension of dimer $\left[\left\{\mathrm{OsCl}(\mu-\mathrm{Cl})\left(\eta^{6}-p \text {-cymene }\right)\right\}_{2}\right](3 ; 0.184 \mathrm{~g}, 0.3 \mathrm{mmol})$ and the corresponding phosphinite ligand $\mathrm{R}_{2} \mathrm{PO}\left(\mathrm{CH}_{2}\right)_{\mathrm{n}} \mathrm{Ph}(\mathbf{1 - 2 a - c} ; 0.8 \mathrm{mmol})$ in $\mathrm{THF}(20 \mathrm{~mL})$ was stirred for $1 \mathrm{~h}$ at room temperature. The resulting solution was then evaporated to dryness, the oily residue formed dissolved in the minimum amount of $\mathrm{CH}_{2} \mathrm{Cl}_{2}(\mathrm{ca} .5 \mathrm{~mL})$, and the product precipitated by adding $20 \mathrm{~mL}$ of a diethyl ether/hexane mixture $(1: 1 \mathrm{v} / \mathrm{v})$. The same precipitation procedure was repeated twice and the yellow solid was finally washed with diethyl ether $(5 \mathrm{~mL})$ and dried in vacuo. Characterization data for the resulting complexes 4-5a-c are as follows.

\subsection{1. $\left[\mathrm{OsCl}_{2}\left(\eta^{6}\right.\right.$-p-cymene) $\left.\left(\mathrm{Ph}_{2} \mathrm{POCH}_{2} \mathrm{Ph}\right)\right]$ (4a)}

Yield: $0.351 \mathrm{~g}(85 \%) .{ }^{31} \mathrm{P}\left\{{ }^{1} \mathrm{H}\right\} \mathrm{NMR}\left(121 \mathrm{MHz}, \mathrm{CDCl}_{3}\right): \delta=64.2$ (s) ppm. ${ }^{1} \mathrm{H}$ NMR $(300 \mathrm{MHz}$, $\left.\mathrm{CDCl}_{3}\right): \delta=8.00-7.93(\mathrm{~m}, 4 \mathrm{H}, \mathrm{Ph}), 7.43-7.28(\mathrm{~m}, 11 \mathrm{H}, \mathrm{Ph}), 5.41$ and $5.35\left(\mathrm{~d}, 2 \mathrm{H}\right.$ each, ${ }^{3} J_{\mathrm{HH}}=5.7 \mathrm{~Hz}$, $\mathrm{CH}$ of $p$-cymene), $4.86\left(\mathrm{~d}, 2 \mathrm{H},{ }^{3} J_{\mathrm{PH}}=5.4 \mathrm{~Hz}, \mathrm{OCH}_{2}\right), 2.54\left(\mathrm{sept}, 1 \mathrm{H},{ }^{3} \mathrm{~J}_{\mathrm{HH}}=6.9 \mathrm{~Hz}, \mathrm{CHMe}\right), 1.96(\mathrm{~s}$, $3 \mathrm{H}, \mathrm{Me}), 1.10\left(\mathrm{~d}, 6 \mathrm{H},{ }^{3} \mathrm{JHH}_{\mathrm{HH}}=6.9 \mathrm{~Hz}, \mathrm{CHMe} 2\right) \mathrm{ppm} .{ }^{13} \mathrm{C}\left\{{ }^{1} \mathrm{H}\right\} \mathrm{NMR}\left(100 \mathrm{MHz}, \mathrm{CDCl}_{3}\right): \delta=137.8(\mathrm{~d}$, ${ }^{3} J_{\mathrm{PC}}=3.5 \mathrm{~Hz}, \mathrm{C}_{\mathrm{ipso}}$ of Ph), $136.2\left(\mathrm{~d},{ }^{1} J_{\mathrm{PC}}=57.0 \mathrm{~Hz}, \mathrm{C}_{\mathrm{ipso}}\right.$ of PPh), $132.6\left(\mathrm{~d}, J_{\mathrm{PC}}=11.0 \mathrm{~Hz}, \mathrm{CH}_{\text {ortho }}\right.$ or $\mathrm{CH}_{\text {meta }}$ of $\left.\mathrm{PPh}\right), 130.9\left(\mathrm{~s}, \mathrm{CH}_{\text {para }}\right.$ of $\left.\mathrm{PPh}\right), 128.4$ and $127.0\left(\mathrm{~s}, \mathrm{CH}_{\text {ortho }}\right.$ and $\mathrm{CH}_{\text {meta }}$ of $\left.\mathrm{Ph}\right), 127.8(\mathrm{~d}$, $J_{\mathrm{PC}}=15.5 \mathrm{~Hz}, \mathrm{CH}_{\text {ortho }}$ or $\mathrm{CH}_{\text {meta }}$ of $\left.\mathrm{PPh}\right), 127.7\left(\mathrm{~s}, \mathrm{CH}_{\text {para }}\right.$ of $\left.\mathrm{Ph}\right), 103.9$ and 91.1 (s, Cof $p$-cymene), 81.8 $\left(\mathrm{d},{ }^{2} J_{\mathrm{PC}}=3.2 \mathrm{~Hz}, \mathrm{CH}\right.$ of $p$-cymene), $80.0\left(\mathrm{~d},{ }^{2} J_{\mathrm{PC}}=4.8 \mathrm{~Hz}, \mathrm{CH}\right.$ of $p$-cymene), $68.2\left(\mathrm{~s}, \mathrm{OCH}_{2}\right), 29.8(\mathrm{~s}$, $\mathrm{CHMe}_{2}$ ), 22.1 (s, $\mathrm{CHMe}$ ), 17.4 (s, Me) ppm. Elemental analysis calcd. (\%) for $\mathrm{C}_{29} \mathrm{H}_{31} \mathrm{Cl}_{2} \mathrm{OPOs}: \mathrm{C} 50.65$, H 4.54; found: C 50.78, H 4.63.

\subsection{2. $\left[\mathrm{OsCl}_{2}\left(\eta^{6}\right.\right.$-p-cymene $\left.)\left(\mathrm{Ph}_{2} \mathrm{POCH}_{2} \mathrm{CH}_{2} \mathrm{Ph}\right)\right](4 \mathbf{b})$}

Yield: $0.299 \mathrm{~g}(71 \%) .{ }^{31} \mathrm{P}\left\{{ }^{1} \mathrm{H}\right\} \mathrm{NMR}\left(121 \mathrm{MHz}, \mathrm{CDCl}_{3}\right): \delta=63.1$ (s) ppm. ${ }^{1} \mathrm{H}$ NMR $(300 \mathrm{MHz}$, $\left.\mathrm{CDCl}_{3}\right): \delta=7.85-7.78(\mathrm{~m}, 4 \mathrm{H}, \mathrm{Ph}), 7.41-7.25(\mathrm{~m}, 11 \mathrm{H}, \mathrm{Ph}), 5.33$ and $5.21\left(\mathrm{~d}, 2 \mathrm{H}\right.$ each, ${ }^{3} J_{\mathrm{HH}}=5.7 \mathrm{~Hz}, \mathrm{CH}$ of $p$-cymene), $3.95\left(\mathrm{td}, 2 \mathrm{H},{ }^{3} J_{\mathrm{HH}}=6.3 \mathrm{~Hz},{ }^{3} \mathrm{JPH}_{\mathrm{PH}}=5.1 \mathrm{~Hz}, \mathrm{OCH}_{2}\right), 2.93\left(\mathrm{t}, 2 \mathrm{H},{ }^{3} J_{\mathrm{HH}}=6.3 \mathrm{~Hz}, \mathrm{CH}_{2} \mathrm{Ph}\right)$, $2.40\left(\mathrm{sept}, 1 \mathrm{H},{ }^{3} \mathrm{~J}_{\mathrm{HH}}=6.9 \mathrm{~Hz}, \mathrm{CHMe}\right), 1.87(\mathrm{~s}, 3 \mathrm{H}, \mathrm{Me}), 1.10\left(\mathrm{~d}, 6 \mathrm{H},{ }^{3} \mathrm{~J}_{\mathrm{HH}}=6.9 \mathrm{~Hz}, \mathrm{CHMe} \mathrm{e}_{2}\right) \mathrm{ppm}$. ${ }^{13} \mathrm{C}\left\{{ }^{1} \mathrm{H}\right\}$ NMR $\left(100 \mathrm{MHz}, \mathrm{CDCl}_{3}\right): \delta=138.7\left(\mathrm{~s}, \mathrm{C}_{\mathrm{ipso}}\right.$ of Ph), $136.3\left(\mathrm{~d},{ }^{1} J_{\mathrm{PC}}=56.2 \mathrm{~Hz}, \mathrm{C}_{\mathrm{ipso}}\right.$ of PPh), 132.5 $\left(\mathrm{d}, J_{\mathrm{PC}}=9.7 \mathrm{~Hz}, \mathrm{CH}_{\text {ortho }}\right.$ or $\mathrm{CH}_{\text {meta }}$ of $\left.\mathrm{PPh}\right), 130.7\left(\mathrm{~s}, \mathrm{CH}_{\text {para }}\right.$ of $\left.\mathrm{PPh}\right), 129.3$ and $128.4\left(\mathrm{~s}, \mathrm{CH}_{\text {ortho }}\right.$ and $\mathrm{CH}_{\text {meta }}$ of $\left.\mathrm{Ph}\right), 127.7\left(\mathrm{~d}, J_{\mathrm{PC}}=8.8 \mathrm{~Hz}, \mathrm{CH}_{\text {ortho }}\right.$ or $\mathrm{CH}_{\text {meta }}$ of $\left.\mathrm{PPh}\right), 126.5\left(\mathrm{~s}, \mathrm{CH}_{\text {para }}\right.$ of $\left.\mathrm{Ph}\right), 102.8$ and 91.7 (s, $\mathrm{C}$ of $p$-cymene), 81.4 and 80.2 (s, $\mathrm{CH}$ of $p$-cymene), $67.6\left(\mathrm{~s}, \mathrm{OCH}_{2}\right), 37.1\left(\mathrm{~s}, \mathrm{CH}_{2} \mathrm{Ph}\right), 29.9\left(\mathrm{~s}, \mathrm{CHMe}_{2}\right)$, 
21.9 (s, $\mathrm{CHMe}$ ), 17.1 (s, Me) ppm. Elemental analysis calcd. (\%) for $\mathrm{C}_{30} \mathrm{H}_{33} \mathrm{Cl}_{2} \mathrm{OPOs}$ : $\mathrm{C} 51.35, \mathrm{H}$ 4.74; found: C 51.26, H 4.97 .

\subsection{3. $\left[\mathrm{OsCl}_{2}\left(\eta^{6}\right.\right.$-p-cymene) $\left.\left(\mathrm{Ph}_{2} \mathrm{POCH}_{2} \mathrm{CH}_{2} \mathrm{CH}_{2} \mathrm{Ph}\right)\right](4 \mathrm{c})$}

Yield: $0.296 \mathrm{~g}(69 \%) .{ }^{31} \mathrm{P}\left\{{ }^{1} \mathrm{H}\right\} \mathrm{NMR}\left(121 \mathrm{MHz}, \mathrm{CD}_{2} \mathrm{Cl}_{2}\right): \delta=62.2$ (s) ppm. ${ }^{1} \mathrm{H}$ NMR $(300 \mathrm{MHz}$, $\left.\mathrm{CD}_{2} \mathrm{Cl}_{2}\right): \delta=7.93-7.87(\mathrm{~m}, 4 \mathrm{H}, \mathrm{Ph}), 7.45-7.18(\mathrm{~m}, 11 \mathrm{H}, \mathrm{Ph}), 5.42$ and $5.33\left(\mathrm{~d}, 2 \mathrm{H}\right.$ each, ${ }^{3} J_{\mathrm{HH}}=5.6 \mathrm{~Hz}, \mathrm{CH}$ of $p$-cymene), $3.82-3.76\left(\mathrm{~m}, 2 \mathrm{H}, \mathrm{OCH}_{2}\right), 2.75\left(\mathrm{t}, 2 \mathrm{H},{ }^{3} J_{\mathrm{HH}}=7.2 \mathrm{~Hz}, \mathrm{CH}_{2} \mathrm{Ph}\right), 2.49\left(\mathrm{sept}, 1 \mathrm{H},{ }^{3} J_{\mathrm{HH}}=6.9 \mathrm{~Hz}\right.$, $\left.\mathrm{CHMe}_{2}\right), 2.01-1.92\left(\mathrm{~m}, 2 \mathrm{H}, \mathrm{CH}_{2}\right), 1.95(\mathrm{~s}, 3 \mathrm{H}, \mathrm{Me}), 1.13\left(\mathrm{~d}, 6 \mathrm{H},{ }^{3} \mathrm{~J}_{\mathrm{HH}}=6.9 \mathrm{~Hz}, \mathrm{CHMe} e_{2}\right) \mathrm{ppm} .{ }^{13} \mathrm{C}\left\{{ }^{1} \mathrm{H}\right\}$ NMR $\left(100 \mathrm{MHz}, \mathrm{CD}_{2} \mathrm{Cl}_{2}\right): \delta=141.5\left(\mathrm{~s}, \mathrm{C}_{\text {ipso }}\right.$ of Ph), $136.5\left(\mathrm{~d},{ }^{1} J_{\mathrm{PC}}=56.7 \mathrm{~Hz}, \mathrm{C}_{\text {ipso }}\right.$ of PPh), $132.5(\mathrm{~d}$, $J_{\mathrm{PC}}=10.8 \mathrm{~Hz}, \mathrm{CH}_{\text {ortho }}$ or $\mathrm{CH}_{\text {meta }}$ of PPh), $130.7\left(\mathrm{~s}, \mathrm{CH}_{\text {para }}\right.$ of PPh), $128.4\left(\mathrm{~s}, \mathrm{CH}_{\text {ortho }}\right.$ and $\mathrm{CH}_{\text {meta }}$ of $\mathrm{Ph}), 127.7\left(\mathrm{~d}, J_{\mathrm{PC}}=10.2 \mathrm{~Hz}, \mathrm{CH}_{\mathrm{ortho}}\right.$ or $\mathrm{CH}_{\text {meta }}$ of PPh), $125.9\left(\mathrm{~s}, \mathrm{CH}_{\text {para }}\right.$ of Ph), 102.8 and $91.2(\mathrm{~s}, \mathrm{C}$ of $p$-cymene), 81.8 (s, CH of $p$-cymene), 80.1 (d, ${ }^{2} J_{\mathrm{PC}}=5.4 \mathrm{~Hz}, \mathrm{CH}$ of $p$-cymene), $66.3\left(\mathrm{~s}, \mathrm{OCH}_{2}\right), 32.1$ (d, $\left.{ }^{3} J_{\mathrm{PC}}=7.9 \mathrm{~Hz}, \mathrm{CH}_{2}\right), 32.0\left(\mathrm{~s}, \mathrm{CH}_{2} \mathrm{Ph}\right), 29.9\left(\mathrm{~s}, \mathrm{CHMe}_{2}\right), 21.9\left(\mathrm{~s}, \mathrm{CHMe} \mathrm{C}_{2}\right), 17.1$ (s, Me) ppm. Elemental analysis calcd. (\%) for $\mathrm{C}_{31} \mathrm{H}_{35} \mathrm{Cl}_{2}$ OPOs: C 59.43, $\mathrm{H} 5.63$; found: C 52.02, $\mathrm{H} 4.93$.

\subsection{4. $\left[\mathrm{OsCl}_{2}\left(\eta^{6}\right.\right.$-p-cymene) $\left.\left({ }^{\mathrm{i}} \mathrm{Pr}_{2} \mathrm{POCH}_{2} \mathrm{Ph}\right)\right](5 \mathbf{a})$}

Yield: $0.249 \mathrm{~g}(67 \%) .{ }^{31} \mathrm{P}\left\{{ }^{1} \mathrm{H}\right\} \mathrm{NMR}\left(121 \mathrm{MHz}, \mathrm{CD}_{2} \mathrm{Cl}_{2}\right): \delta=103.5$ (s) ppm. ${ }^{1} \mathrm{H} \mathrm{NMR}(300 \mathrm{MHz}$, $\left.\mathrm{CD}_{2} \mathrm{Cl}_{2}\right): \delta=7.45-7.38(\mathrm{~m}, 5 \mathrm{H}, \mathrm{Ph}), 5.62$ and $5.55\left(\mathrm{~d}, 2 \mathrm{H}\right.$ each, ${ }^{3} \mathrm{~J}_{\mathrm{HH}}=5.9 \mathrm{~Hz}, \mathrm{CH}$ of $p$-cymene), 5.02 $\left(\mathrm{d}, 2 \mathrm{H},{ }^{3} J_{\mathrm{PH}}=4.2 \mathrm{~Hz}, \mathrm{OCH}_{2}\right), 3.15-2.98(\mathrm{~m}, 2 \mathrm{H}, \mathrm{PCHMe}), 2.74\left(\mathrm{sept}, 1 \mathrm{H},{ }^{3} \mathrm{~J}_{\mathrm{PH}}=6.9 \mathrm{~Hz}, \mathrm{CHMe}_{2}\right)$, $2.17(\mathrm{~s}, \mathrm{Me}), 1.43\left(\mathrm{dd}, 6 \mathrm{H},{ }^{3} J_{\mathrm{PH}}=13.8 \mathrm{~Hz},{ }^{3} J_{\mathrm{HH}}=7.2 \mathrm{~Hz}, \mathrm{PCHMe} e_{2}\right), 1.31\left(\mathrm{dd}, 6 \mathrm{H},{ }^{3} J_{\mathrm{PH}}=15.3 \mathrm{~Hz}\right.$, $\left.{ }^{3} J_{\mathrm{HH}}=7.2 \mathrm{~Hz}, \mathrm{PCHMe} e_{2}\right), 1.20\left(\mathrm{~d}, 6 \mathrm{H},{ }^{3} J_{\mathrm{HH}}=6.9 \mathrm{~Hz}, \mathrm{CHMe} e_{2}\right) \mathrm{ppm} .{ }^{13} \mathrm{C}\left\{{ }^{1} \mathrm{H}\right\} \mathrm{NMR}\left(100 \mathrm{MHz}, \mathrm{CD}_{2} \mathrm{Cl}_{2}\right)$ : $\delta=137.7\left(\mathrm{~d},{ }^{3} J_{\mathrm{PC}}=6.4 \mathrm{~Hz}, \mathrm{C}_{\mathrm{ipso}}\right.$ of $\left.\mathrm{Ph}\right), 128.6$ and $127.5\left(\mathrm{~s}, \mathrm{CH}_{\text {ortho }}\right.$ and $\mathrm{CH}_{\text {meta }}$ of $\left.\mathrm{Ph}\right), 128.1\left(\mathrm{~s}, \mathrm{CH}_{\text {para }}\right.$ of Ph), 98.8 and 91.1 (s, C of $p$-cymene), $80.5\left(\mathrm{~d},{ }^{2} J_{\mathrm{PC}}=3.8 \mathrm{~Hz}, \mathrm{CH}\right.$ of $p$-cymene), $80.3\left(\mathrm{~d},{ }^{2} J_{\mathrm{PC}}=3.6 \mathrm{~Hz}\right.$, $\mathrm{CH}$ of $p$-cymene), $69.4\left(\mathrm{~d},{ }^{2} J_{\mathrm{PC}}=10.2 \mathrm{~Hz}, \mathrm{OCH}_{2}\right), 29.8\left(\mathrm{~s}, \mathrm{CHMe}_{2}\right), 28.5\left(\mathrm{~d},{ }^{1} J_{\mathrm{PC}}=27.0 \mathrm{~Hz}, \mathrm{PCHMe}_{2}\right)$, $22.1\left(\mathrm{~s}, \mathrm{CHMe} e_{2}\right), 17.5$ and 17.1 (s, PCHMe $), 17.4$ (s, Me) ppm. Elemental analysis calcd. (\%) for $\mathrm{C}_{23} \mathrm{H}_{35} \mathrm{Cl}_{2} \mathrm{OPOs:} \mathrm{C} 44.58$, H 5.69; found: C 44.80, H 5.80.

\subsection{5. $\left[\mathrm{OsCl}_{2}\left(\eta^{6}\right.\right.$-p-cymene) $\left.\left({ }^{\mathrm{i}} \mathrm{Pr}_{2} \mathrm{POCH}_{2} \mathrm{CH}_{2} \mathrm{Ph}\right)\right](5 \mathbf{b})$}

Yield: $0.277 \mathrm{~g}(73 \%) .{ }^{31} \mathrm{P}\left\{{ }^{1} \mathrm{H}\right\} \mathrm{NMR}\left(121 \mathrm{MHz}, \mathrm{CD}_{2} \mathrm{Cl}_{2}\right): \delta=103.3$ (s) ppm. ${ }^{1} \mathrm{H} \mathrm{NMR}(300 \mathrm{MHz}$, $\left.\mathrm{CD}_{2} \mathrm{Cl}_{2}\right): \delta=7.41-7.29(\mathrm{~m}, 5 \mathrm{H}, \mathrm{Ph}), 5.50$ and $5.44\left(\mathrm{~d}, 2 \mathrm{H}\right.$ each, ${ }^{3} J_{\mathrm{HH}}=5.8 \mathrm{~Hz}, \mathrm{CH}$ of $p$-cymene), $4.24\left(\mathrm{td}, 2 \mathrm{H},{ }^{3} J_{\mathrm{HH}}=6.3 \mathrm{~Hz},{ }^{3} J_{\mathrm{PH}}=3.9 \mathrm{~Hz}, \mathrm{OCH}_{2}\right), 3.04\left(\mathrm{t}, 2 \mathrm{H},{ }^{3} J_{\mathrm{HH}}=6.3 \mathrm{~Hz}, \mathrm{CH}_{2} \mathrm{Ph}\right), 2.99-2.89$ $(\mathrm{m}, 2 \mathrm{H}, \mathrm{PCHMe}), 2.74\left(\mathrm{sept}, 1 \mathrm{H},{ }^{3} J_{\mathrm{PH}}=6.8 \mathrm{~Hz}, \mathrm{CHMe}\right), 2.14(\mathrm{~s}, \mathrm{Me}), 1.35\left(\mathrm{dd}, 6 \mathrm{H},{ }^{3} J_{\mathrm{PH}}=13.8 \mathrm{~Hz}\right.$, $\left.{ }^{3} \mathrm{~J}_{\mathrm{HH}}=7.2 \mathrm{~Hz}, \mathrm{PCHMe} 2\right), 1.24-1.17(\mathrm{~m}, 12 \mathrm{H}, \mathrm{PCHMe}$ and PCHMe $)$ ppm. ${ }^{13} \mathrm{C}\left\{{ }^{1} \mathrm{H}\right\} \mathrm{NMR}(100 \mathrm{MHz}$, $\left.\mathrm{CD}_{2} \mathrm{Cl}_{2}\right): \delta=138.1$ (s, $\mathrm{C}_{\text {ipso }}$ of $\left.\mathrm{Ph}\right), 128.9$ and $128.6\left(\mathrm{~s}, \mathrm{CH}_{\text {ortho }}\right.$ and $\mathrm{CH}_{\text {meta }}$ of $\left.\mathrm{Ph}\right), 126.7\left(\mathrm{~s}, \mathrm{CH}_{\text {para }}\right.$ of $\mathrm{Ph}$ ), 97.8 and 89.5 (s, C of $p$-cymene), 81.1 (d, ${ }^{2} J_{\mathrm{PC}}=3.5 \mathrm{~Hz}, \mathrm{CH}$ of $p$-cymene), $80.6\left(\mathrm{~d},{ }^{2} J_{\mathrm{PC}}=3.4 \mathrm{~Hz}\right.$, $\mathrm{CH}$ of $p$-cymene), $68.7\left(\mathrm{~d},{ }^{2} J_{\mathrm{PC}}=12.0 \mathrm{~Hz}, \mathrm{OCH}_{2}\right), 37.4\left(\mathrm{~d},{ }^{3} \mathrm{~J}_{\mathrm{PC}}=5.9 \mathrm{~Hz}, \mathrm{CH}_{2} \mathrm{Ph}\right), 29.9\left(\mathrm{~s}, \mathrm{CHMe}_{2}\right), 27.6$ $\left(\mathrm{d},{ }^{1} J_{\mathrm{PC}}=26.4 \mathrm{~Hz}, \mathrm{PCHMe}_{2}\right), 22.2\left(\mathrm{~s}, \mathrm{CHMe}_{2}\right), 17.3(\mathrm{~s}, \mathrm{PCHMe}), 16.7(\mathrm{~s}, \mathrm{Me}) \mathrm{ppm}$. Elemental analysis calcd. (\%) for $\mathrm{C}_{24} \mathrm{H}_{37} \mathrm{Cl}_{2} \mathrm{OPOs:} \mathrm{C} 45.49$, $\mathrm{H} 5.89$; found: C 45.65, $\mathrm{H} 5.96$.

\subsection{6. $\left[\mathrm{OsCl}_{2}\left(\eta^{6}\right.\right.$-p-cymene) $\left.\left.{ }^{\mathrm{i}} \mathrm{Pr}_{2} \mathrm{POCH}_{2} \mathrm{CH}_{2} \mathrm{CH}_{2} \mathrm{Ph}\right)\right](5 \mathrm{c})$}

Yield: $0.307 \mathrm{~g}(79 \%) .{ }^{31} \mathrm{P}\left\{{ }^{1} \mathrm{H}\right\} \mathrm{NMR}\left(121 \mathrm{MHz}, \mathrm{CDCl}_{3}\right): \delta=102.3$ (s) ppm. ${ }^{1} \mathrm{H} \mathrm{NMR}\left(\mathrm{CDCl}_{3}\right)$ : $\delta=7.37-7.22(\mathrm{~m}, 5 \mathrm{H}, \mathrm{Ph}), 5.67$ and $5.59\left(\mathrm{~d}, 2 \mathrm{H}\right.$ each, ${ }^{3} J_{\mathrm{HH}}=5.8 \mathrm{~Hz}, \mathrm{CH}$ of $p$-cymene $), 4.01-3.96(\mathrm{~m}$, $\left.2 \mathrm{H}, \mathrm{OCH}_{2}\right), 3.00-2.90\left(\mathrm{~m}, 3 \mathrm{H}, \mathrm{CHMe}_{2}\right.$ and $\left.\mathrm{PCHMe}_{2}\right), 2.79\left(\mathrm{t}, 2 \mathrm{H},{ }^{3} \mathrm{~J}_{\mathrm{HH}}=7.5 \mathrm{~Hz}, \mathrm{CH}_{2} \mathrm{Ph}\right), 2.21(\mathrm{~s}, \mathrm{Me})$, 2.09-2.03 (m, 2H, CH $), 1.38-1.23\left(\mathrm{~m}, 18 \mathrm{H}, \mathrm{CHMe}_{2}\right.$ and $\left.\mathrm{PCHMe} e_{2}\right) \mathrm{ppm} .{ }^{13} \mathrm{C}\left\{{ }^{1} \mathrm{H}\right\} \mathrm{NMR}(100 \mathrm{MHz}$, $\left.\mathrm{CDCl}_{3}\right): \delta=141.0\left(\mathrm{~s}, \mathrm{C}_{\text {ipso }}\right.$ of $\left.\mathrm{Ph}\right), 128.6$ and $128.2\left(\mathrm{~s}, \mathrm{CH}_{\text {ortho }}\right.$ and $\mathrm{CH}_{\text {meta }}$ of $\left.\mathrm{Ph}\right), 126.2\left(\mathrm{~s}, \mathrm{CH}_{\text {para }}\right.$ of $\mathrm{Ph}), 99.1$ and 90.0 (s, C of $p$-cymene), $80.7\left(\mathrm{~d},{ }^{2} J_{\mathrm{PC}}=4.0 \mathrm{~Hz}, \mathrm{CH}\right.$ of $p$-cymene), $80.3\left(\mathrm{~d},{ }^{2} J_{\mathrm{PC}}=3.9 \mathrm{~Hz}\right.$, $\mathrm{CH}$ of $p$-cymene), $66.9\left(\mathrm{~d},{ }^{2} J_{\mathrm{PC}}=11.0 \mathrm{~Hz}, \mathrm{OCH}_{2}\right), 32.8\left(\mathrm{~d},{ }^{3} J_{\mathrm{PC}}=6.0 \mathrm{~Hz}, \mathrm{CH}_{2}\right), 32.2\left(\mathrm{~s}, \mathrm{CH}_{2} \mathrm{Ph}\right), 29.9(\mathrm{~s}$, $\left.\mathrm{CHMe}_{2}\right), 27.9\left(\mathrm{~d},{ }^{1} \mathrm{~J}_{\mathrm{PC}}=26.8 \mathrm{~Hz}, \mathrm{PCHMe}_{2}\right), 22.5\left(\mathrm{~s}, \mathrm{CHMe} e_{2}\right), 17.7(\mathrm{~s}, \mathrm{Me}), 17.6$ and $17.1\left(\mathrm{~s}, \mathrm{PCHMe}_{2}\right)$, ppm. Elemental analysis calcd. (\%) for $\mathrm{C}_{25} \mathrm{H}_{39} \mathrm{Cl}_{2}$ OPOs: C 46.36, $\mathrm{H}$ 6.07; found: $\mathrm{C} 46.23, \mathrm{H} 6.01$. 


\subsection{Preparation of Complex $\left[\mathrm{OsCl}_{2}\left(\eta^{6}\right.\right.$-benzene $\left.)\left\{{ }^{i} \mathrm{Pr}_{2} \mathrm{PO}\left(\mathrm{CH}_{2}\right)_{2} \mathrm{Ph}\right\}\right]$ (7)}

A suspension of dimer $\left[\left\{\mathrm{OsCl}(\mu-\mathrm{Cl})\left(\eta^{6} \text {-benzene }\right)\right\}_{2}\right](6 ; 0.203 \mathrm{~g}, 0.3 \mathrm{mmol})$ and the phosphinite ligand ${ }^{i} \mathrm{Pr}_{2} \mathrm{PO}\left(\mathrm{CH}_{2}\right)_{2} \mathrm{Ph}(\mathbf{2} \mathbf{b} ; 0.191 \mathrm{~g}, 0.8 \mathrm{mmol})$ in toluene $(20 \mathrm{~mL})$ was heated under refluxing conditions for $24 \mathrm{~h}$. The reaction mixture was then filtered over Kieselguhr and the filtrate evaporated to dryness. The resulting oily residue was then dissolved in the minimum amount of $\mathrm{CH}_{2} \mathrm{Cl}_{2}(\sim 5 \mathrm{~mL})$, and the product precipitated by adding $20 \mathrm{~mL}$ of hexane. The same precipitation procedure was repeated twice and the yellow solid was finally washed with hexane $(5 \mathrm{~mL})$ and dried in vacuo. Yield: $0.149 \mathrm{~g}(43 \%)$. ${ }^{31} \mathrm{P}\left\{{ }^{1} \mathrm{H}\right\}$ NMR (121 MHz, $\left.\mathrm{CD}_{2} \mathrm{Cl}_{2}\right): \delta=101.0$ (s) ppm. ${ }^{1} \mathrm{H}$ NMR $\left(300 \mathrm{MHz}, \mathrm{CD}_{2} \mathrm{Cl}_{2}\right): \delta=7.38-7.25(\mathrm{~m}$, $5 \mathrm{H}, \mathrm{Ph}), 5.64\left(\mathrm{~s}, 6 \mathrm{H}, \mathrm{C}_{6} \mathrm{H}_{6}\right), 4.44\left(\mathrm{td}, 2 \mathrm{H},{ }^{3} J_{\mathrm{HH}}=6.3 \mathrm{~Hz},{ }^{3} J_{\mathrm{PH}}=3.9 \mathrm{~Hz}, \mathrm{OCH}_{2}\right), 2.99\left(\mathrm{t}, 2 \mathrm{H},{ }^{3} J_{\mathrm{HH}}=6.3 \mathrm{~Hz}\right.$, $\left.\mathrm{CH}_{2} \mathrm{Ph}\right), 2.95-2.87(\mathrm{~m}, 2 \mathrm{H}, \mathrm{PCHMe} 2), 1.30\left(\mathrm{dd}, 6 \mathrm{H},{ }^{3} J_{\mathrm{PH}}=14.7 \mathrm{~Hz},{ }^{3} J_{\mathrm{HH}}=7.2 \mathrm{~Hz}, \mathrm{PCHMe}\right), 1.20(\mathrm{dd}$, $\left.\left.6 \mathrm{H},{ }^{3} J_{\mathrm{PH}}=15.0 \mathrm{~Hz},{ }^{3} J_{\mathrm{HH}}=7.2 \mathrm{~Hz}, \mathrm{PCHMe} e_{2}\right) \mathrm{ppm} .{ }^{13} \mathrm{C}^{1} \mathrm{H}\right\} \mathrm{NMR}\left(100 \mathrm{MHz}, \mathrm{CD}_{2} \mathrm{Cl}_{2}\right): \delta=138.6(\mathrm{~s}$, $\mathrm{C}_{\text {ipso }}$ of $\left.\mathrm{Ph}\right), 129.1$ and 128.5 (s, $\mathrm{CH}_{\text {ortho }}$ and $\mathrm{CH}_{\text {meta }}$ of $\left.\mathrm{Ph}\right), 126.6\left(\mathrm{~s}, \mathrm{CH}_{\text {para }}\right.$ of $\left.\mathrm{Ph}\right), 80.5\left(\mathrm{~s}, \mathrm{C}_{6} \mathrm{H}_{6}\right), 68.2$ $\left(\mathrm{d},{ }^{2} J_{\mathrm{PC}}=8.6 \mathrm{~Hz}, \mathrm{OCH}_{2}\right), 37.2\left(\mathrm{~d},{ }^{3} J_{\mathrm{PC}}=6.8 \mathrm{~Hz}, \mathrm{CH}_{2} \mathrm{Ph}\right), 28.8\left(\mathrm{~d},{ }^{1} J_{\mathrm{PC}}=29.9 \mathrm{~Hz}, \mathrm{PCHMe}_{2}\right), 17.4(\mathrm{~d}$, ${ }^{2} J_{\mathrm{PC}}=4.8 \mathrm{~Hz}, \mathrm{PCHMe} 2$ ) ppm. Elemental analysis calcd. (\%) for $\mathrm{C}_{20} \mathrm{H}_{29} \mathrm{Cl}_{2} \mathrm{OPOs}: \mathrm{C} 41.59, \mathrm{H}$ 5.06; found: C 41.74, H 4.99 .

\subsection{X-ray Crystal Structure Determination of Compound 4c}

Crystals of $4 \mathbf{c}$ suitable for X-ray diffraction analysis were obtained by slow diffusion of hexane into a saturated solution of the complex in dichloromethane. The most relevant crystal and refinement data are collected in Table 2. Diffraction data were recorded on an Oxford Diffraction Xcalibur Nova single-crystal diffractometer using $\mathrm{Cu}-\mathrm{K} \alpha$ radiation $(\lambda=1.5418 \AA$ ). Images were collected at a fixed crystal-to-detector distance of $62 \mathrm{~mm}$ using the oscillation method with $1.10^{\circ}$ oscillation and $1.25-2.5 \mathrm{~s}$ variable exposure time per image. Data collection strategy was calculated with the program CrysAlis Pro CCD [24]. Data reduction and cell refinement were performed with the program CrysAlis Pro RED [24], and an empirical absorption correction was applied by means of a SCALE3 ABSPACK algorithm as implemented in the program CrysAlis Pro RED [24]. The software package WINGX was used for space group determination, structure solution, and refinement [25]. The structure was solved by Paterson interpretation and phase expansion using DIRDIF2008 [26]. Isotropic least-squares refinement on $F^{2}$ using SHELXL97 was performed [27].

During the final stages of the refinements, all the positional parameters and the anisotropic temperature factors of all non- $\mathrm{H}$ atoms were refined except $\mathrm{C}(8), \mathrm{C}(9 \mathrm{~A}), \mathrm{C}(9 \mathrm{~B}), \mathrm{C}(10 \mathrm{~A})$, and $\mathrm{C}(10 \mathrm{~B})$, because the isopropyl group of the $p$-cymene ligand was found in two disordered positions $(69 \%$ for the major position and $31 \%$ for the minor one). The distances from $C(9 \mathrm{~A}), \mathrm{C}(9 \mathrm{~B}), \mathrm{C}(10 \mathrm{~A})$, and $\mathrm{C}(10 \mathrm{~B})$ to $\mathrm{C}(8)$ were fixed at $1.50 \AA$ (by comparison with other structures previously described $[14,15])$. All $\mathrm{H}$ atoms were geometrically located and their coordinates were refined riding on their parent atoms. The function minimized was $\left[\Sigma w\left(F_{\mathrm{o}}{ }^{2}-{F_{\mathrm{c}}}^{2}\right) / \Sigma w\left(F_{\mathrm{o}}{ }^{2}\right)\right]^{1 / 3}$ where $\mathrm{w}=1 /\left[\sigma^{2}\left(F_{\mathrm{o}}{ }^{2}\right)+(0.0460 P)^{2}+6.2548 P\right]$ with $\sigma\left(F_{\mathrm{o}}{ }^{2}\right)$ from counting statistics and $P=\left(\operatorname{Max}\left(F_{\mathrm{o}}{ }^{2}, 0\right)+2 F_{\mathrm{c}}{ }^{2}\right) / 3$. Atomic scattering factors were taken from the International Tables for X-ray Crystallography [28]. Geometrical calculations related to the centroid $C^{*}$ were made with PARST [29]. CCDC-1972510 contains the supplementary crystallographic data for this paper. These data can be obtained free of charge via http://www.ccdc.cam.ac.uk/conts/retrieving.html (or from the CCDC, 12 Union Road, Cambridge CB2 1EZ, UK; Fax: +44 1223 336033; E-mail: deposit@ccdc.cam.ac.uk). 
Table 2. Crystal data and structure refinement details for compound 4c.

\begin{tabular}{|c|c|}
\hline Chemical Formula & $\mathrm{C}_{31} \mathrm{H}_{35} \mathrm{Cl}_{2} \mathrm{OOsP}$ \\
\hline fw & 715.66 \\
\hline $\mathrm{T}(\mathrm{K})$ & 293(2) \\
\hline cryst. syst. & monoclinic \\
\hline space group & $\mathrm{P} 21 / \mathrm{c}$ \\
\hline cryst. size $\mathrm{mm}^{3}$ & $0.24 \times 0.16 \times 0.16$ \\
\hline$a, \AA$ & $10.3465(2)$ \\
\hline$b, \AA$ & 19.1010(3) \\
\hline$c, \AA$ & $15.1007(3)$ \\
\hline$\alpha, \operatorname{deg}$ & 90 \\
\hline$\beta, \operatorname{deg}$ & $105.009(2)$ \\
\hline$\gamma, \operatorname{deg}$ & 90 \\
\hline Z & 4 \\
\hline$V, \AA^{3}$ & $2882.5(1)$ \\
\hline$\rho_{\text {calcd }}, \mathrm{g} \mathrm{cm}^{-3}$ & 1.649 \\
\hline$\mu, \mathrm{mm}^{-1}$ & 10.751 \\
\hline $\mathrm{F}(000)$ & 1416 \\
\hline$\theta$ range, deg & 3.813 to 69.621 \\
\hline index ranges & $-12 \leq \mathrm{h} \leq 12 ;-22 \leq \mathrm{k} \leq 22 ;-18 \leq 1 \leq 13$ \\
\hline completeness to $\theta_{\max }$ & $97.9 \%$ \\
\hline no. of data collected & 14414 \\
\hline no. of unique data & 5321 \\
\hline no. of parameters/restrains & $319 / 4$ \\
\hline refinement method & full-matrix least-squares on $F^{2}$ \\
\hline goodness of fit on $\mathrm{F}^{2}$ & 1.065 \\
\hline $\mathrm{R} 1^{a}[\mathrm{I}>2 \sigma(\mathrm{I})]$ & 0.0407 \\
\hline $\mathrm{wR}^{a}{ }^{a}[\mathrm{I}>2 \sigma(\mathrm{I})]$ & 0.0978 \\
\hline R1 (all data) & 0.0486 \\
\hline wR2 (all data) & 0.1033 \\
\hline largest diff. peak and hole, e $\AA^{-3}$ & 0.935 and -1.683 \\
\hline
\end{tabular}

\section{Conclusions}

In summary, different half-sandwich arene-Os(II) complexes bearing $P$-coordinated phosphinite ligands, namely, $\left[\mathrm{OsCl}_{2}\left(\eta^{6}\right.\right.$-p-cymene $\left.)\left\{\mathrm{R}_{2} \mathrm{PO}\left(\mathrm{CH}_{2}\right)_{n} \mathrm{Ph}\right\}\right]\left(\mathrm{R}=\mathrm{Ph}, \mathrm{n}=1(\mathbf{4 a}), 2(\mathbf{4 b}), 3\right.$ (4c); $\mathrm{R}={ }^{\mathrm{i}} \mathrm{Pr}$, $\mathrm{n}=1(\mathbf{5 a}), 2(\mathbf{5 b}), 3(\mathbf{5 c}))$ and $\left[\mathrm{OsCl}_{2}\left(\eta^{6}\right.\right.$-benzene) $\left.\left.{ }_{\{}^{\mathrm{i}} \mathrm{Pr}_{2} \mathrm{PO}\left(\mathrm{CH}_{2}\right)_{2} \mathrm{Ph}\right\}\right](7)$, have been synthesized and characterized. Unlike their ruthenium analogs, these compounds are completely inert and do not undergo intramolecular exchange of the $\eta^{6}$-coordinated arene ligand under heating or MW-irradiation, thus preventing the access to tethered species featuring a $\eta^{6}: \kappa^{1}(P)$-coordination of the phosphinites.

Supplementary Materials: The following are available online, Figures S1-S21: NMR spectra of the osmium complexes 4-5a-c and 7.

Author Contributions: Conceptualization, V.C. and P.C.; Synthesis and spectroscopic characterization of the complexes, R.G.-F.; Crystal structure refinement, J.B.; All the authors contributed to the discussion of the experimental results as well as writing and editing of the manuscript. All authors have read and agreed to the published version of the manuscript.

Funding: This research was funded by the Spanish Ministry of Economy, Industry and Competitiveness (MINECO project CTQ2016-75986-P) and the University of Oviedo (project PAPI-18-GR-2011-0032).

Acknowledgments: R.G.-F. thanks MECD of Spain for the award of a FPU fellowship.

Conflicts of Interest: The authors declare no conflicts of interest. 


\section{References}

1. González-Fernández, R.; Crochet, P.; Cadierno, V. Half-sandwich ruthenium(II) complexes with tethered arene-phosphinite ligands: Synthesis, structure and application in catalytic cross dehydrogenative coupling reactions of silanes and alcohols. Dalton Trans. 2020, 49, 210-222. [CrossRef]

2. Miyaki, Y.; Onishi, T.; Kurosawa, H. Synthesis and reaction of ruthenium(II) complexes containing heteroatom donor $(\mathrm{O}, \mathrm{N}, \mathrm{P})$ tethered to $\eta^{6}$-arene ring. Inorg. Chim. Acta 2000, 300-302, 369-377. [CrossRef]

3. Weber, I.; Heinemann, F.W.; Bauer, W.; Superchi, S.; Zahl, A.; Richter, D.; Zenneck, U. Diastereoselective routes to $\left[\right.$ Amino $\left\{\sigma(P): \eta^{6}\right.$-(ansa-phosphinite)benzene $\}$ chlororuthenium(II) $] \mathrm{PF}_{6}$ salts: Kinetic versus thermodynamic preferences. Organometallics 2008, 27, 4116-4125. [CrossRef]

4. Pandey, M.K.; Mague, J.T.; Balakrishna, M.S. Sterically demanding phosphines with 2,6-dibenzhydryl-4-methylphenyl core: Synthesis of $\mathrm{Ru}^{\mathrm{II}}, \mathrm{Pd}^{\mathrm{II}}$, and $\mathrm{Pt}^{\mathrm{II}}$ complexes, and structural and catalytic studies. Inorg. Chem. 2018, 57, 7468-7480. [CrossRef]

5. Bennett, M.A.; Harper, J.R. Tethered arene complexes of ruthenium. In Modern Coordination Chemistry; Leigh, G.J., Winterton, N., Eds.; RSC Publishing: Cambridge, UK, 2002; pp. 163-168.

6. Clark, T.J.; Lee, K.; Manners, I. Transition-metal-catalyzed dehydrocoupling: A convenient route to bonds between main-group elements. Chem. Eur. J. 2006, 12, 8634-8648. [CrossRef]

7. Kuciński, K.; Hreczycho, G. Catalytic formation of silicon-heteroatom (N, P, O, S) bonds. ChemCatChem 2017, 9, 1868-1885. [CrossRef]

8. Ventura-Espinosa, D.; Sabater, S.; Carretero-Cerdán, A.; Baya, M.; Mata, J.A. High production of hydrogen on demand from silanes catalyzed by iridium complexes as a versatile hydrogen storage system. ACS Catal. 2018, 8, 2558-2566. [CrossRef]

9. Esteruelas, M.A.; López, A.M.; Oñate, E.; Royo, E. Synthesis and reactivity of osmium complexes containing a cyclopentadienyl ligand with a pendant phosphine donor group. Organometallics 2004, 23, 3021-3030. [CrossRef]

10. Batuecas, M.; Esteruelas, M.A.; García-Yebra, C.; Oñate, E. Redox isomerization of allylic alcohols catalyzed by osmium and ruthenium complexes containing a cyclopentadienyl ligand with a pendant amine or phosphoramidite group: X-ray structure of an $\eta^{3}$-1-hydroxyallyl-metal-hydride intermediate. Organometallics 2010, 29, 2166-2175. [CrossRef]

11. Baya, M.; Crochet, P.; Esteruelas, M.A.; Oñate, E. Generation of functionally substituted cyclopentadienyl ligands in osmium(IV) chemistry. Organometallics 2001, 20, 240-253. [CrossRef]

12. Braunschweig, H.; Dellermann, T.; Dewhurst, R.D.; Mies, J.; Radacki, K.; Stellwag-Konertz, S.; Vargas, A. Strained ansa half-sandwich complexes of ruthenium and osmium and a non-iron metallopolymer by ring-opening polymerization. Organometallics 2014, 33, 1536-1539. [CrossRef]

13. Zhou, X.; He, X.; Lin, J.; Zhuo, Q.; Chen, Z.; Zhang, H.; Wang, J.; Xia, H. Reactions of osmium hydrido alkenylcarbyne with allenoates: Insertion and [3 + 2] annulation. Organometallics 2015, 34, 1742-1750. [CrossRef]

14. Bell, A.G.; Koźmiński, W.; Linden, A.; von Philipsborn, W. ${ }^{187}$ Os NMR study of ( $\eta^{6}$-arene)osmium(II) complexes: Separation of electronic and steric ligand effects. Organometallics 1996, 15, 3124-3135. [CrossRef]

15. González-Fernández, R.; Crochet, P.; Cadierno, V.; Menéndez, M.I.; López, R. Phosphinous acid-assisted hydration of nitriles: Understanding the controversial reactivity of osmium and ruthenium catalysts. Chem. Eur. J. 2017, 23, 15210-15221. [CrossRef]

16. Freedman, D.A.; Magneson, D.J.; Mann, K.R. Synthesis, characterization, and photochemistry of bis(arene) and bis(acetonitrile) complexes of osmium(II). Inorg. Chem. 1995, 34, 2617-2624. [CrossRef]

17. Freedman, D.A.; Gill, T.P.; Blough, A.M.; Koefod, R.S.; Mann, K.R. Preparation and reactions of $\left[\mathrm{CpOs}\left(\mathrm{CH}_{3} \mathrm{CN}\right)_{3}\right]^{+}$. A useful synthetic intermediate for the preparation of $\mathrm{CpOsL}_{3}$ compounds. Inorg. Chem. 1997, 36, 95-102. [CrossRef]

18. Wang, R.; Eberspacher, T.A.; Hasegawa, T.; Day, V.; Ware, D.C.; Taube, H. Tmtacn, tacn, and triammine complexes of $\left(\eta^{6}\right.$-arene)Os ${ }^{\mathrm{II}}$ : Syntheses, characterizations, and photosubstitution reactions (tmtacn =1,4,7-trimethyl-1,4,7-triazacyclononane; tacn =1,4,7-triazacyclononane). Inorg. Chem. 2001, 40, 593-600. [CrossRef]

19. Armarego, W.L.F.; Chai, C.L.L. Purification of Laboratory Chemicals, 5th ed.; Butterworth-Heinemann: Oxford, UK, 2003. 
20. Masutani, K.; Minowa, T.; Hagiwara, Y.; Mukaiyama, T. Cyanation of alcohols with diethyl cyanophosphonate and 2,6-dimethyl-1,4-benzoquinone by a new type of oxidation-reduction condensation. Bull. Chem. Soc. Jpn. 2006, 79, 1106-1117. [CrossRef]

21. Shintou, T.; Kikuchi, W.; Mukaiyama, T. Efficient method for the preparation of carboxylic acid alkyl esters or alkyl phenyl ethers by a new type of oxidation-reduction condensation using 2,6-dimethyl-1,4-benzoquinone and alkoxydiphenylphosphines. Bull. Chem. Soc. Jpn. 2003, 76, 1645-1667. [CrossRef]

22. Castarlenas, R.; Esteruelas, M.A.; Oñate, E. N-Heterocyclic carbene-osmium complexes for olefin metathesis reactions. Organometallics 2005, 24, 4343-4346. [CrossRef]

23. Arthur, T.; Stephenson, T.A. Synthesis of triple halide-bridged arene complexes of ruthenium(II) and osmium(II). J. Organomet. Chem. 1981, 208, 369-387. [CrossRef]

24. CrysAlis ${ }^{\text {Pro }}$ CCD \& CrysAlis ${ }^{\text {Pro }}$ RED; Oxford Diffraction Ltd.: Oxford, UK, 2008.

25. Farrugia, L.J. WinGX and ORTEP for Windows: An update. J. Appl. Cryst. 2012, 45, 849-854. [CrossRef]

26. Beurskens, P.T.; Beurskens, G.; de Gelder, R.; García-Granda, S.; Gould, R.O.; Smits, J.M.M. DIRDIF2008 Program System. Crystallography Laboratory; University of Nijmegen: Nijmegen, The Netherlands, 2008.

27. Sheldrick, G.M. SHELXL97: Program for the Refinement of Crystal Structures; University of Göttingen: Göttingen, Germany, 1997.

28. Wilson, A.J.C. (Ed.) International Tables for X-ray Crystallography, Volume, C; Kluwer Academic Publishers: Dordrecht, The Netherlands, 1992.

29. Nardelli, M. PARST: A system of FORTRAN routines for calculating molecular structure parameters from results of crystal structure analyses. Comput. Chem. 1983, 7, 95-98. [CrossRef]

(C) 2020 by the authors. Licensee MDPI, Basel, Switzerland. This article is an open access article distributed under the terms and conditions of the Creative Commons Attribution (CC BY) license (http://creativecommons.org/licenses/by/4.0/). 